\title{
DIAGNOSTIC SIGNIFICANCE OF MEASURING RETICULOCYTE MATURITY INDICES IN IRON DEFICIENCY ANAEMIA
}

\author{
Tanweer Ahmed, Asad Mahmood, Nasir Uddin*, Helen Mary Robert, Rafia Mahmood, Usman Tahir Swati \\ Armed Forces Institute of Pathology/National University of Medical Sciences (NUMS) Rawalpindi Pakistan, *Combined Military Hospital Lahore/ \\ National University of Medical Sciences (NUMS) Pakistan
}

\begin{abstract}
Objective: To evaluate the diagnostic significance of reticulocyte maturity indices in iron deficiency anaemia.

Study Design: Comparative cross-sectional study.

Place and Duration of Study: Department of Hematology, Armed Forces Institute of Pathology, Rawalpindi, from Sep 2019 to Jun 2020.

Methodology: A total of 340 children, ages between 1 and 5 years of either gender, were divided into two groups based on haemoglobin and ferritin level. Group A comprised of 203 children with haemoglobin $>11 \mathrm{~g} / \mathrm{dL}$ andferritin level $>7 \mathrm{ng} /$ $\mathrm{mL}$. Group B comprised of 137 children with haemoglobin $<11 \mathrm{~g} / \mathrm{dL}$ and ferritin $<7 \mathrm{ng} / \mathrm{mL}$. Red blood cell and reticulocyte parameters were compared.

Results: Iron deficiency anaemia was found to be $37.6 \%$ in the study population. Comparison revealed that the children in groups B had significantly higher mean red cell distribution width $(17.1 \pm 1.3 \%$ vs. $13.3 \pm 1.3 \%)$ and significantly lower hemoglobin $(9.5 \pm 1.0 \mathrm{~g} / \mathrm{dL}$ vs. $12.7 \pm 1.0 \mathrm{~g} / \mathrm{dL})$, mean corpuscular hemoglobin $(21.8 \pm 2.5 \mathrm{pg}$ vs. $28.6 \pm 2.3 \mathrm{pg})$, mean corpuscular volume $(69.4 \pm 3.5 \mathrm{fL}$ vs. $80.4 \pm 3.3 \mathrm{fL})$, mean corpuscular hemoglobin concentration $(31.5 \pm 1.8 \mathrm{~g} / \mathrm{dL}$ vs. $32.7 \pm 1.6$ $\mathrm{g} / \mathrm{dL})$ and serum ferritin level $(4.5 \pm 2.6 \mathrm{mg} / \mathrm{mL}$ vs. $55.9 \pm 32.1 \mathrm{ng} / \mathrm{mL})$. No significant differences in the mean total red blood cell count, hematocrit and reticulocytes between two groups were noted $(p>0.05)$ and significant differences were noted in terms of low, medium \& high fluorescence ratios in both groups $(p<0.05)$.

Conclusion: Our findings suggest that medium fluorescence ratio and high fluorescence ratio were higher among children with iron deficiency anaemia.
\end{abstract}

Keywords: High fluorescence ratio, Iron deficiency anaemia, Medium fluorescence ratio, Reticulocyte maturity indices.

How to Cite This Article: Ahmed T, Mahmood A, Uddin N, Robert HM, Mahmood R, Swati UT. Diagnostic Significance of Measuring Reticulocyte Maturity Indices in Iron Deficiency Anaemia. Pak Armed Forces Med J 2021; 71(6): 2109-2113. Doi: https://doi.org/10.51253/pafmj.v71i6.5642

\footnotetext{
This is an Open Access article distributed under the terms of the Creative Commons Attribution License (https://creativecommons.org/licenses/by-nc/4.0/), which permits unrestricted use, distribution, and reproduction in any medium, provided the original work is properly cited.
}

\section{INTRODUCTION}

Anaemia is described as hemoglobin $(\mathrm{Hb})$ level below normal but the criteria vary according to age and gender. Among children under the age of 5 years, "A secondary analysis of National Nutrition Survey Data 2011-2012" published in 2016, revealed prevalence of iron deficiency anaemia as 33.2\%. ${ }^{1}$ As per "World Health Organization (WHO)" in children of 6 months to 4 years, anaemia is labeled as hemoglobin less than or equal to $11 \mathrm{~g} / \mathrm{dL} .^{2}$ Peripheral blood reticulocyte count is considered helpful in diagnosis, classification and evaluation of individuals with anaemia while it also holds the center stage for evaluation and bone marrow response to anaemia treatment. ${ }^{3}$

Iron deficiency anaemia (IDA) is among the top 5 leading causes of years spent with disability. ${ }^{4}$ Not only IDA is a major concern in pediatric age groups,

Correspondence: Dr Tanweer Ahmed, Resident Haematology, Combined Military Hospital Lahore Pakistan

Received: 06 Nov 2020; revision received: 12 Feb 2021; accepted: 20 Feb 2021 pre-menopausal and pregnant ladies, but it is also considered a clinical issue affecting individuals who present with different medical and surgical morbidities. ${ }^{5}$ It needs prompt and vigilant evaluation for the diagnosis and management of IDA to improve wellbeing of the affected patients. For identifying high-risk population, early detection of anaemia and evaluation of response to therapy, new laboratory parameters are being studied. ${ }^{6}$ These new parameters are only available in modern automated hematology analyzers that are costly and not available at many places in developing countries like Pakistan. These new parameters are also not part of routine lab reports at the moment due to lack of standardization and studies on large number of population samples.

Reticulocytes are known to be non-nucleated immature red blood cells (RBCs) in the peripheral blood and contain reticulo-filamentous material composed of residual ribonucleic acid (RNA). ${ }^{7}$ For reticulocyte counts, manual method is mostly adopted in diagnostic haematology laboratories. RNA has the propensity of 
reacting with certain supravital stains like New Methylene Blue or Brilliant Cresyl Blue. This results in formation of blue to purple granular or filamentous precipitate that can be viewed under microscope. Such reticulocytes are counted under high power field and reported as percentage of red blood cells. ${ }^{8}$ This manual method is inherently inferior to newer automated reticulocyte analysis as it is time consuming and requires manual labor, skillful technician and microscopist. This method also does not provide any information about newer reticulocyte parameters.

Recently, Automated Reticulocyte Analysis (ARA) using flow cytometry is commonly used as a substitute to manual counts. ARA is considered a more rapid and accurate method which is easy to perform and provides number of reticulocytes count as well as numerous other indices that can help in diagnosing various pathologies and also in monitoring of bone marrow recovery. ${ }^{9}$ Many modern automated hematology analyzers are equipped with ARA. As reticulocyte indices need standardization and labeling of reference values, their regular and proper use in clinical settings is yet to be seen. With ARA, reticulocytes can be classified into three subpopulations as per fluorescence intensity, reflecting maturity i.e. more the RNA content and fluorescence intensity, less mature the reticulocytes. These subpopulations or indices are Low Fluorescence Ratio (LFR), Medium Fluorescence Ratio (MFR) and High Fluorescence Ratio (HFR). With this classification, intracellular RNA levels are directly linked to fluorescence intensity which show degree of maturation of reticulocytes. ${ }^{10}$

Newer parameters related to reticulocytes have not been researched in our setup. International studies have demonstrated promising research in this regard and have suggested the routine use of these parameters for evaluation of anemias including IDA. Keeping this in view, this study was planned to evaluate diagnostic significance of measuring LFR, MFR and HFR in children aged 1-5 years with IDA.

\section{METHODOLOGY}

This comparative cross-sectional study was conducted at Department of Hematology, Armed Forces Institute of Pathology, Rawalpindi, from September 2019 ot June 2020. Approval from Institutional Review Board (FC-HEM18-1/READ-IRB/20/571 Dated 23 Aug 2019) was obtained before starting this study. Using non-probability consecutive sampling technique, a sample size of 340 participants was calculated with $33 \%$ prevalence of IDA in our population of interest and confidence interval of $95 \%$.

Inclusion Criteria: Children of either gender with age 1-5 years were included in the study.

Exclusion Criteria: Children taking any iron or vitamin supplements (as confirmed by parents/guardians of the children) were excluded.

All the study participants were divided into 2 groups. Group A $(n=203)$ comprised of children with $\mathrm{Hb}>11 \mathrm{~g} / \mathrm{dL}$ and serum ferritin level $>7 \mathrm{ng} / \mathrm{mL}$. Group B (n=137) comprised of children with $\mathrm{Hb}<11$ $\mathrm{g} / \mathrm{dL}$ and serum ferritin level $<7 \mathrm{ng} / \mathrm{mL}$. The purpose of study and procedures were explained and informed consent was sought from parents/guardians of all the study participants.

Venous whole blood samples from all children were drawn in commercially available Ethylenediaminetetraacetic acid (EDTA) containing whole blood collection tube (for complete blood counts and reticulocyte parameters) and Gel tube (for serum ferritin level). Samples were properly labelled and dealt within 3 hours of collection. For every blood sample from the participants, the hematology analysis was performed on a "Sysmex XN-3000 automated hematology analyzer" while serum ferritin level was measured using a Chemiluminescence-based automated IMMULITE 1000 immunoassay analyzer. Reticulocyte count was corrected by using following formula, keeping normal hematocrit at $45 \%$ : corrected reticulocyte count $(\%)=$ (Patient's Hematocrit/40) x (Reticulocyte count \%).

Children having anaemia (group B) were compared with those who did not have anaemia (group A). All the data was processed through IBM Statistical Package for Social Sciences (SPSS), version 26. Mean and standard deviations of were calculated for quantitative variables including parameters related to red blood cells and reticulocytes. Independent sample ttest used to compare groups considering $p$-value of $\leq 0.05$ as statistically significant.

\section{RESULTS}

Out of 340 participants of the study, 184 (54.1\%) were males and $156(45.9 \%)$ were females. The mean age at the time of study was 1.59 years. Frequency of IDA in the population under study was noted to be $128(37.6 \%)$. Comparison of red blood cell parameters between the two groups was shown in Table-I. When compared to group A, the children in groups B had significantly higher mean red cell distribution width (RDW\%) and significantly lower haemoglobin (g/dl), 
mean corpuscular haemoglobin $(\mathrm{MCH})$, mean corpuscular volume (MCV), mean corpuscular haemoglo-bin concentration (MCHC) and serum ferritin levels. No statistically significant difference in mean total red blood cell count (TRBC) $(p=0.08)$ and Haematocrit (HCT) $(p=0.09)$ between the two groups was noted.

Table-I: Red blood cell parameters and serum ferritin in both groups .

\begin{tabular}{l|c|c|c}
\hline Parameters & $\begin{array}{c}\text { Group A } \\
(\mathbf{n}=\mathbf{2 0 3})\end{array}$ & $\begin{array}{c}\text { Group B } \\
(\mathbf{n = 1 3 7 )}\end{array}$ & $\begin{array}{c}p \text { - } \\
\text { value }\end{array}$ \\
\hline $\begin{array}{l}\text { Total Red Blood Cell Count } \\
\text { (TRBC, } \times 109 / \mathrm{L})\end{array}$ & $4.7 \pm 2.6$ & $4.3 \pm 0.4$ & 0.080 \\
\hline Hemoglobin (Hb, g/dL) & $12.7 \pm 1.0$ & $9.5 \pm 1.0$ & $<0.001$ \\
\hline Hematocrit (Hct, \%) & $36.4 \pm 4.0$ & $35.6 \pm 3.9$ & 0.090 \\
\hline $\begin{array}{l}\text { Mean corpuscular hemoglobin } \\
\text { (MCH, pg) }\end{array}$ & $28.6 \pm 2.3$ & $21.8 \pm 2.5$ & $<0.001$ \\
\hline $\begin{array}{l}\text { Mean Corpuscular Volume } \\
\text { (MCV, fL) }\end{array}$ & $80.4 \pm 3.3$ & $69.4 \pm 3.5$ & $<0.001$ \\
\hline $\begin{array}{l}\text { Mean Corpuscular } \\
\text { Hemoglobin concentration } \\
\text { (MCHC, g/dL) }\end{array}$ & $32.7 \pm 1.6$ & $31.5 \pm 1.8$ & $<0.001$ \\
\hline $\begin{array}{l}\text { Red Cell Distribution width } \\
\text { (RDW, \%) }\end{array}$ & $13.3 \pm 1.3$ & $17.1 \pm 1.3$ & $<0.001$ \\
\hline Serum Ferritin (ng/mL) & $55.9 \pm 32.1$ & $4.5 \pm 2.6$ & $<0.001$ \\
\hline
\end{tabular}

Table-II shows comparison of reticulocyte parameters between the two groups. There was no statistical difference in mean reticulocytes (\%) between group A and group B $(p=0.100)$. In terms of reticulocyte indices, significant difference was observed in LFR, MFR and HRF $(p<0.05)$ between two groups.

Table-II: Reticulocyte parameters in both groups.

\begin{tabular}{l|c|c|c}
\hline Parameters & $\begin{array}{c}\text { Group A } \\
(\mathbf{n = 2 0 3 )}\end{array}$ & $\begin{array}{c}\text { Group B } \\
(\mathbf{n = 1 3 7 )}\end{array}$ & $\begin{array}{c}p- \\
\text { value }\end{array}$ \\
\hline Reticulocytes (\%) & $1.1 \pm 0.3$ & $1.1 \pm 0.3$ & 0.100 \\
\hline $\begin{array}{l}\text { Low Fluorescence Ratio } \\
\text { (LFR, \% total reticulocytes) }\end{array}$ & $91.9 \pm 3.7$ & $85.9 \pm 4.0$ & $<0.001$ \\
\hline $\begin{array}{l}\text { Medium Fluorescence Ratio } \\
\text { (MFR, \% total reticulocytes) }\end{array}$ & $7.0 \pm 3.6$ & $11.2 \pm 3.9$ & $<0.001$ \\
\hline $\begin{array}{l}\text { High Fluorescence Ratio } \\
\text { (HFR, \% total reticulocytes) }\end{array}$ & $1.0 \pm 0.7$ & $2.8 \pm 0.8$ & $<0.001$ \\
\hline
\end{tabular}

\section{DISCUSSION}

While traversing reticulocyte channel in Sysmex automated hematology analyzers, cell membranes of red blood cells (RBCs), white blood cells (WBCs) and platelets are perforated by a specific lysing reagent. A fluorescent marker then penetrates these cells and tags the nucleic acids in cytoplasm and nuclei. Intensity of fluorescent signal is directly proportional to the amount of nucleic acids in these cells. This is followed by separation of reticulocytes from mature RBCs. Reti-culocyte maturity indices (LFR, MFR \&and
HFR) are also measured. This is based on the flow cytometric prin-ciples of forward scatter and fluorescent intensity. ${ }^{11}$ These indices are known to highlight reticulocyte im-maturity and bone marrow activity.

This study has been carried out with an aim to evaluate reticulocyte maturity indices among children with and without IDA. We measured these reticulocyte maturity indices with a modern and automated state of the art Sysmex XN-3000 Hematology Analyzer and found statistically significant difference in terms of reticulocyte maturity indices between patients with IDA and those without IDA. It seems that monitoring of effective erythropoiesis in response to anaemia can be assessed employing quantitative measurements of reticulocyte subpopulations. Our findings highlighted that evaluating reticulocyte maturity indices using quantification of fractions of reticulocytes as LFR, MFR or HFR can be performed easily where facilities of resources and newer automated hematology analyzers exist. Reticulocyte fluorescence intensity has been found to be directly linked with the quantity of intracellular ribonucleic acid. ${ }^{12}$ Immature reticulocytes fraction (IRF) is the sum of medium fluorescence ration (MFR) and high florescence ratio (HFR). IRF indicates young reticulocytes released into peripheral circulation prematurely and these parameters can be effectively used for the evaluation of erythropoietic efficiency during anaemias including IDA. ${ }^{13,14}$

A possible explanation for the findings of our study is that a rise in the immature reticulocytes in the blood of a patient with IDA significantly highlights body's response to iron deficiency anaemia provided that the medullary tissues and cortical elements needed for erythropoiesis cycle are conserved. Anaemic hypoxia is also thought to stimulate release of erythropoietin from the kidneys which results in increased cell proliferation and differentiation. ${ }^{15}$ This explanation also highlights that the presence of functioning erythropoiesis cycle along with erythropoietin's response to anaemic hypoxia can be ensured by measuring reticulocyte maturity indices and IRF during initial evaluation and subsequent monitoring of the response to therapy.

Maturation time of reticulocytes might decrease in bone marrow in case of severe anaemia, ${ }^{16}$ as higher quantity of immature reticulocytes are discharged in the peripheral blood and stay there for an estimated more than 48 hours until these get transformed into mature RBCs, hence, number of immature reticulocy- 
tes in the peripheral blood is increased. ${ }^{17}$ Indices linked to immaturity of reticulocytes are increased in IDA demonstrating a decline in raw materials required for the production of hemoglobin and transformation of reticulocytes into mature RBCs. Findings of our study are in agreement with other international studies which reported increase in MFR and HFR in patients with anaemias including IDA. Sunkara et al, demonstrated that diagnosis of IDA can be made helpful if various reticulocyte parameters are adopted without using any further biochemical investigations. The study found that, owing to ineffective erythropoiesis, immature reticulocyte fraction and immature reticulocyte subpopulations were significantly higher in IDA. ${ }^{18}$ Wollmann et al, demonstrated that reticulocyte indices related to maturity may be utilized as early markers of IDA and anemia based on their findings that in comparison to control group, individuals suffering from IDA presented with an increased proportion of mean fluorescence ration $(10.3 \pm 4.7 \%$ vs. $6.0 \pm 3.4 \%$; $p$-value $=$ $0.003)$ and high fluorescence ratio $(2.3 \pm 0.87 \%$ vs. $0.9 \pm$ $0.9 \%$; $p$-value $=0.03) .{ }^{19}$ Choi et al, compared reticulocyte maturity indices \& iron-related parameters between healthy and iron deficient females. The study demonstrated that middle \& high fluorescence ratios started increasing when serum iron and serum ferritin levels were depleted. The increment in reticulocyte maturity indices was greatest when iron deficiency was overt. ${ }^{14}$ Zhao et al, compared various red blood cell \& reticulocyte parameters during evaluation of anemias and found that the low fluorescent reticulocyte sub-population significantly decreased, while, medium \& high fluorescent reticulate subpopulations increased in all cases of anemia compared to control population. The study suggested utilizing mean corpuscular volume, red cell distribution width and reticulocyte maturityrelated parameters for the evaluation of various anemias. ${ }^{20}$ Velasco-Rodríguez et al, analyzed various reticulocyte parameters in delta-beta thalassemia trait, beta thalassemia trait and IDA and correlated them with the corresponding pathophysiologic features. The study demonstrated that patients with IDA had much more immature reticulocytes and less absolute reticulocyte count than beta thalassemia trait. The study suggested that not only red cell indices but also reticulocyte maturity indices may be utilized to differentiate the three clinical entities. ${ }^{21}$

Since average lifecycle of an $\mathrm{RBC}$ in peripheral circulation is about 120 days, the usual renewal of RBC mass equates with $1 \%$ of RBCs in circulation, therefore, identification of abnormality in the routine hematimet- ric indices of patients with IDA may require weeks-tomonths. ${ }^{12,18}$ On the other hand, reticulocyte maturity indices show changes much earlier with the benefits of automation and more accuracy. For these reasons, reticulocyte maturity indices can prove helpful as significant and early markers of anemias including IDA.

\section{LIMITATION Of STUDY}

Limitations in the usage of reticulocyte maturity indices in the laboratory practices at some facilities, because of either definitions or issues due to standardization and correlation between different methods employed, could pose a significant challenge. The findings of this study warrant further research involving different age groups with larger sample size of both healthy individuals and population at high risk of developing IDA at different centers of Pakistan so that benefits of automation and newer reticulocyte parameters may be reaped by inducting these tests in routine catalogues of haematology laboratories.

\section{CONCLUSION}

Our findings suggest that MFR and HFR are high among children with IDA, highlighting increased erythropoietic activity. These indices can be adopted as rapid, less time consuming, less labor intensive and lesser invasive procedure for early diagnosis of iron deficiency anaemia.

\section{Conflict of Interest: None.}

\section{Authors' Contribution}

TA: Direct contribution to conception, desing, analysis \& interpretation, AM: Intellectual contribution to analysis, literature review \& manuscript preparation, NU: Intellectual contribution to analysis, literature review \& manuscript preparation, HMR: Intellectual contribution to analysis, literature review \& manuscript preparation, RM: Intellectual contribution, manuscript preparation, UTS: Manuscript preparation \& data analysis.

\section{REFERENCES}

1. Habib MA, Black K, Soofi SB, Hussain I, Bhatti Z, Bhutta ZA, et al. Prevalence and predictors of iron deficiency anaemia in children under five years of age in pakistan, a secondary analysis of national nutrition survey Data 2011-2012. PLoS One 2016; 11(5): e0155051.

2. Leal LP, Batista Filho M, Lira PI, Figueiroa JN, Osório MM. Prevalence of anemia and associated factors in children aged 659 months in Pernambuco, Northeastern Brazil. Rev Saude Publica 2011; 45(3): 457-466.

3. Buttarello M. Laboratory diagnosis of anaemia: are the old and new red cell parameters useful in classification and treatment, how?. Int J Lab Hematol 2016; 38(Suppl-1): 123-132.

4. Mawani M, Ali SA, Bano G, Ali SA. Iron deficiency anaemia among women of reproductive age, an important public health problem: Situation analysis. Reprod Syst Sex Disord 2016; 5(3): 1000187.

5. Cappellini MD, Musallam KM, Taher AT. Iron deficiency anaemia revisited. J Intern Med 2020; 287(2): 153-170.

6. Means RT. Iron deficiency and iron deficiency anaemia: implications and impact in pregnancy, fetal development, and early childhood parameters. Nutrients 2020; 12(2): 447. 


\section{Reticulocyte Maturity Indices}

7. Chandran R,Mohan P. Temporal trends in reticulocyte count with Methylene blue staining: A comparative study to assess reliability of reticulocyte count as a quality control parameter. Int J Clin Diagn Pathol 2020; 3(1): 371-375.

8. Moradabadi A, Khaleghi M, Shahdoost M, Farsinejad A. optimized method for reticulocyte counting: simple, accurate, and comparable to flow cytometry. Iran J Ped Hematol Oncol 2019; 9(1): 17-24.

9. Komal P, Siddhi P. Comparison of automated flowcytometric reticulocyte analysis with manual reticulocyte count. Int J Res Med Sci 2019; 7(10): 3825.

10. Urrechaga E, Borque L, Escanero JF. Erythrocyte and reticulocyte parameters in iron deficiency and thalassemia. J Clin Lab Anal 2011; 25(3): 223-228.

11. Seed Haematology - The importance of reticulocyte detection. Sysmex Education Enhancement and Development. 2016. [Internet] Available from: https://www.sysmexeurope.com/fileadmin/media/f100/SEED/Sysmex_SEED_The_ importance_of_reticulocyte_detection.pdf (Accessed on August 11, 2019)

12. João AR, Pinto S, Costa E. Reticulocyte subpopulations and immature reticulocyte fractions as indicators of increased erythropoiesis in patients with iron deficiency anaemia. Rev Bras Hematol Hemoter 2007; 30(3): 188-192.

13. Buttarello M. Laboratory diagnosis of anaemia: are the old and new red cell parameters useful in classification and treatment, how?. Int J Lab Hematol 2016; 38(Suppl-1): 123-132.
14. Choi JW, Pai SH. Reticulocyte subpopulations and reticulocyte maturity index (RMI) rise as body iron status fall. Am J Hematol. 2001; 67(2): 130-135.

15. Lesesve JF, Lacombe F, Marit G. High fluorescence reticulocytes are an indicator of bone marrow recovery after chemotherapy. Eur J Haematol 1995; 54(1): 61-63.

16. Agarwal AM, Rets A. Laboratory approach to investigation of anaemia with a focus on pyruvate kinase deficiency. Int J Lab Hematol 2020; 42(Suppl-1): 107-112.

17. Thomas C, Thomas L. Biochemical markers and hematologic indices in the diagnosis of functional iron deficiency. Clin Chem 2002; 48(7): 1066-1076.

18. Sunkara A, Kotta DR. Evaluation of red cell indices and reticulocyte maturity indices including reticulocyte haemoglobin concentration in iron deficiency anaemia in adult female population. J Evid Based Med Healthc 2016; 97(3): 5315-5318. Wollmann M, Gerzson BM, Schwert V, Figuera RW, RitzelGde O. Reticulocyte maturity indices in iron deficiency anaemia. Rev Bras Hematol Hemoter 2014; 36(1): 25-28.

Zhao L, Mao ZG, Jiang H, Qin L, Huang CY, Tan B. Value of $\mathrm{MCV} / \mathrm{RDW}$ combined with reticulocyte parameters in differential diagnosis of anemia diseases. Zhongguo Shi Yan Xue Ye Xue Za Zhi (Chinese) 2015; 23(6): 1662-1666.

19. Velasco-Rodríguez D, Alonso-Domínguez JM, González-Fernández FA. Reticulocyte parameters of delta beta thalassaemia trait, beta thalassaemia trait and iron deficiency anaemia. J Clin Pathol 2016; 69(2): 149-154. 